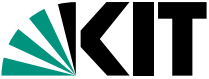

Karlsruhe Institute of Technology
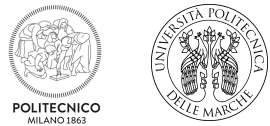

\title{
Production, transport and dissipation of turbulent stresses across scales and space
}

Davide Gatti, A. Chiarini, A. Cimarelli, M. Quadrio | September 6, 2018

INTERNATIONAL TURBULENCE INITIATIVE , 5 - 7 SEPTEMBER 2018, BERTINORO, ITALY

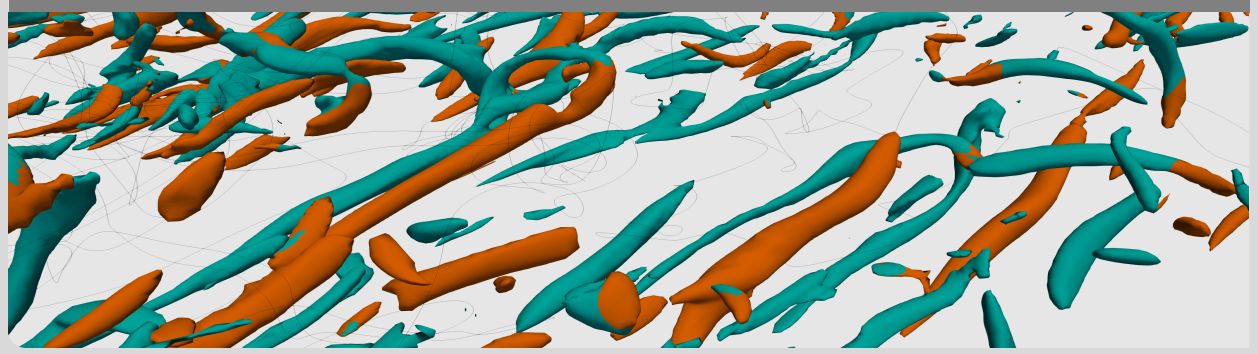




\section{(Two) Classic approaches to turbulence}

Space of scales
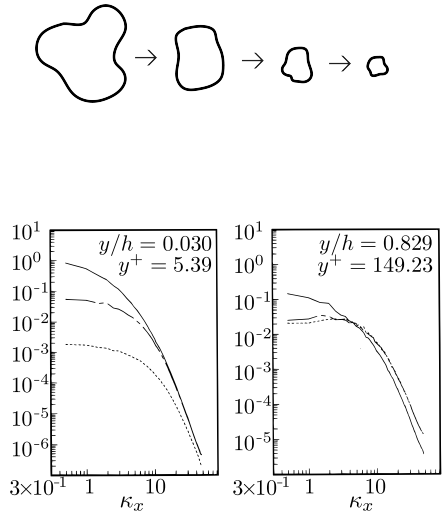

Kim et al. JFM 1987

\section{Physical space}

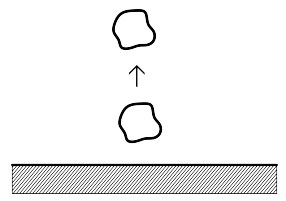

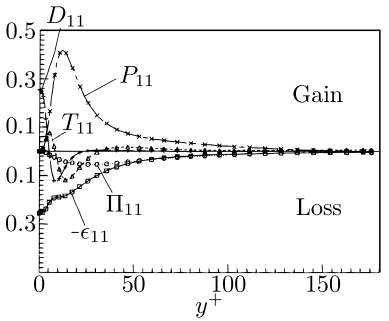

Mansour et al. JFM 1988

AGKE

OO

$-00$

D. Gatti - Production, transport and dissipation of Reynolds stresses
Example results

0000

Conclusion

000

September 6, 2018 


\section{Generalized Kolmogorov Equation (GKE)}

Exact budget equation for $\left\langle\delta u_{i} \delta u_{i}\right\rangle=\langle\delta u \delta u\rangle+\langle\delta v \delta v\rangle+\langle\delta w \delta w\rangle$ 


\section{Generalized Kolmogorov Equation (GKE)}

Exact budget equation for $\left\langle\delta u_{i} \delta u_{i}\right\rangle=\langle\delta u \delta u\rangle+\langle\delta v \delta v\rangle+\langle\delta w \delta w\rangle$

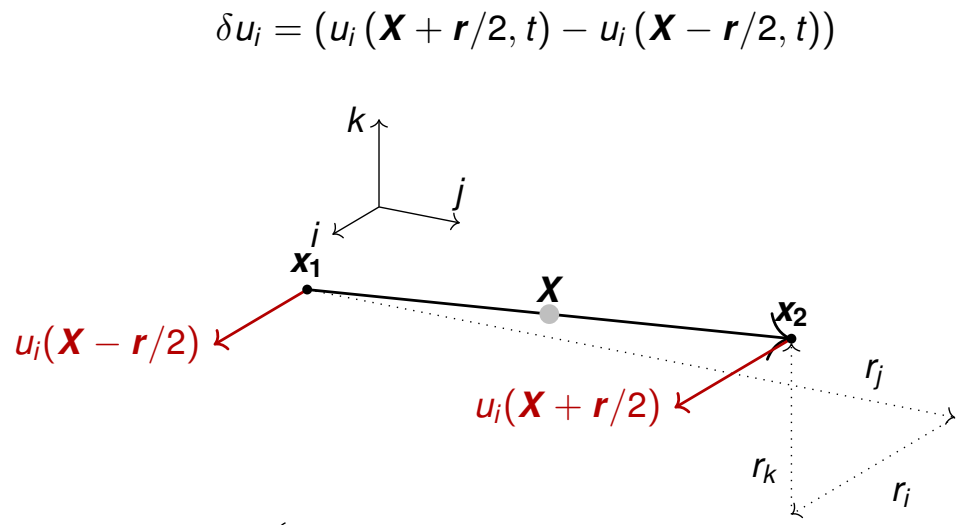

dependent on:

$$
\begin{aligned}
& X=\left(x_{1}+x_{2}\right) / 2 \\
& r=x_{2}-x_{1}
\end{aligned}
$$




\section{Generalized Kolmogorov Equation (GKE)}

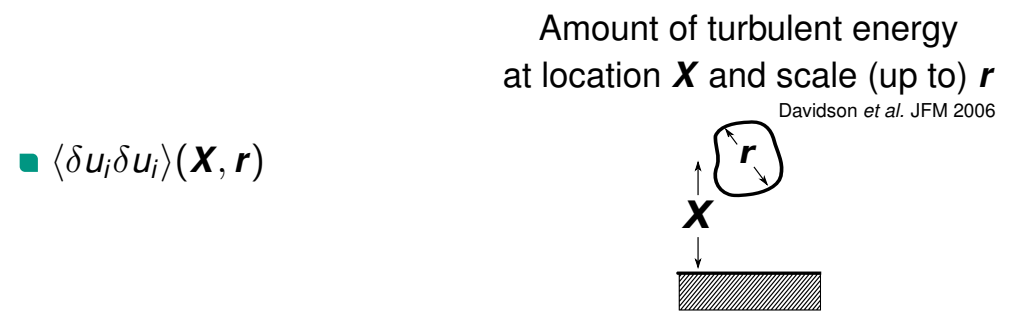




\section{Generalized Kolmogorov Equation (GKE)}

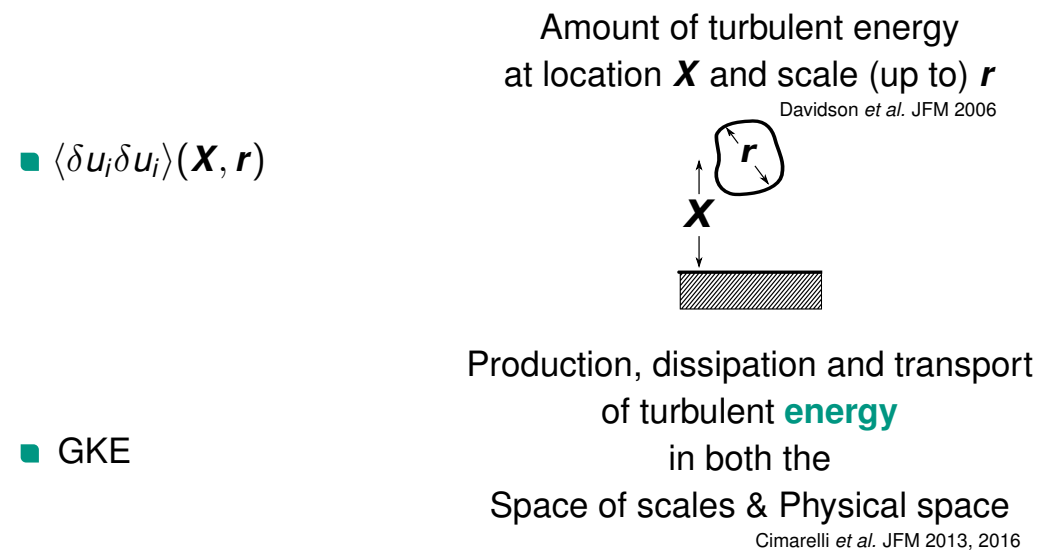




\section{Generalized Kolmogorov Equation (GKE)}

- $\left\langle\delta u_{i} \delta u_{i}\right\rangle(\boldsymbol{X}, \boldsymbol{r})$

- GKE

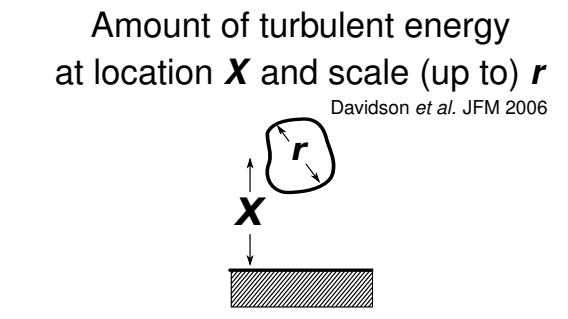

Production, dissipation and transport of turbulent energy

in both the

Space of scales \& Physical space

Cimarelli et al. JFM 2013, 2016

$\left\langle\delta u_{i} \delta u_{i}\right\rangle=\langle\delta u \delta u\rangle+\langle\delta v \delta v\rangle+\langle\delta w \delta w\rangle \rightarrow$...anisotropy? 


\section{GKE: budget for $\left\langle\delta u_{i} \delta u_{i}\right\rangle$}

$$
\begin{aligned}
& \frac{\partial \phi_{k}}{\partial r_{k}}+\frac{\partial \psi_{k}}{\partial X_{k}}=\xi \\
& \text { scale flux } \phi_{k}=\underbrace{\delta u_{k}\left\langle\delta u_{i} \delta u_{i}\right\rangle}_{\text {mean transport }}+\underbrace{\left\langle\delta u_{k} \delta u_{i} \delta u_{i}\right\rangle}_{\text {turbulent transport }} \underbrace{-2 \nu \frac{\partial}{\partial r_{k}}\left\langle\delta u_{i} \delta u_{i}\right\rangle}_{\text {viscous diffusion }} \\
& \text { space flux } \psi_{k}=\underbrace{\left\langle u_{k}^{*} \delta u_{i} \delta u_{i}\right\rangle}_{\text {turbulent transport }}+\underbrace{\frac{1}{\rho}\left\langle\delta p \delta u_{i}\right\rangle}_{\text {pressure transport }} \underbrace{-\frac{\nu}{2} \frac{\partial}{\partial X_{k}}\left\langle\delta u_{i} \delta u_{i}\right\rangle}_{\text {viscous diffusion }} \\
& \text { source } \xi=\underbrace{-2\left\langle u_{k}{ }^{*} \delta u_{i}\right\rangle \delta\left(\frac{\partial U_{i}}{\partial x_{k}}\right)}_{\text {production }} \underbrace{-2\left\langle\delta u_{k} \delta u_{i}\right\rangle\left(\frac{\partial U_{i}}{\partial x_{k}}\right)^{*}}_{\text {production }} \underbrace{-4 \epsilon_{i i}{ }^{*}}_{\text {dissipation }}
\end{aligned}
$$




\section{Anisotropic GKEs (AGKEs): budget for $\left\langle\delta u_{i} \delta u_{j}\right\rangle$}

$$
\frac{\partial \phi_{k, i j}}{\partial r_{k}}+\frac{\partial \psi_{k, i j}}{\partial X_{k}}=\xi_{i j}
$$

scale flux $\phi_{k, i j}=\underbrace{\delta u_{k}\left\langle\delta u_{i} \delta u_{j}\right\rangle}_{\text {mean transport }}+\underbrace{\left\langle\delta u_{k} \delta u_{i} \delta u_{j}\right\rangle}_{\text {turbulent transport }} \underbrace{-2 \nu \frac{\partial}{\partial r_{k}}\left\langle\delta u_{i} \delta u_{j}\right\rangle}_{\text {viscous diffusion }}$

space flux $\psi_{k, i j}=\underbrace{\left\langle u_{k}^{*} \delta u_{i} \delta u_{j}\right\rangle}_{\text {turbulent transport }}+\underbrace{\frac{1}{\rho}\left\langle\delta p \delta u_{i}\right\rangle \delta_{k j}+\frac{1}{\rho}\left\langle\delta p \delta u_{j}\right\rangle \delta_{k i}}_{\text {pressure transport }} \underbrace{-\frac{\nu}{2} \frac{\partial}{\partial X_{k}}\left\langle\delta u_{i} \delta u_{j}\right\rangle}_{\text {viscous diffusion }}$

$$
\text { source } \xi_{i j}=\underbrace{-\left\langle u_{k}{ }^{*} \delta u_{j}\right\rangle \delta\left(\frac{\partial U_{i}}{\partial x_{k}}\right)-\left\langle u_{k}{ }^{*} \delta u_{i}\right\rangle \delta\left(\frac{\partial U_{j}}{\partial x_{k}}\right)}+
$$

production

$\underbrace{-\left\langle\delta u_{k} \delta u_{j}\right\rangle\left(\frac{\partial U_{i}}{\partial x_{k}}\right)^{*}-\left\langle\delta u_{k} \delta u_{i}\right\rangle\left(\frac{\partial U_{j}}{\partial x_{k}}\right)^{*}}+\underbrace{\frac{1}{\rho}\left\langle\delta p \frac{\partial \delta u_{i}}{\partial X_{j}}\right\rangle+\frac{1}{\rho}\left\langle\delta p \frac{\partial \delta u_{j}}{\partial X_{i}}\right\rangle} \underbrace{-4 \epsilon_{i j}^{*}}$

production

Introduction

000

AGKE

0

Example results

0000

dissipation 


\section{AGKEs for indefinite plane channels}

$$
\left\langle\delta u_{i} \delta u_{j}\right\rangle(\boldsymbol{X}, \boldsymbol{r}) \rightarrow\left\langle\delta u_{i} \delta u_{j}\right\rangle\left(Y, r_{x}, r_{y}, r_{z}\right)
$$

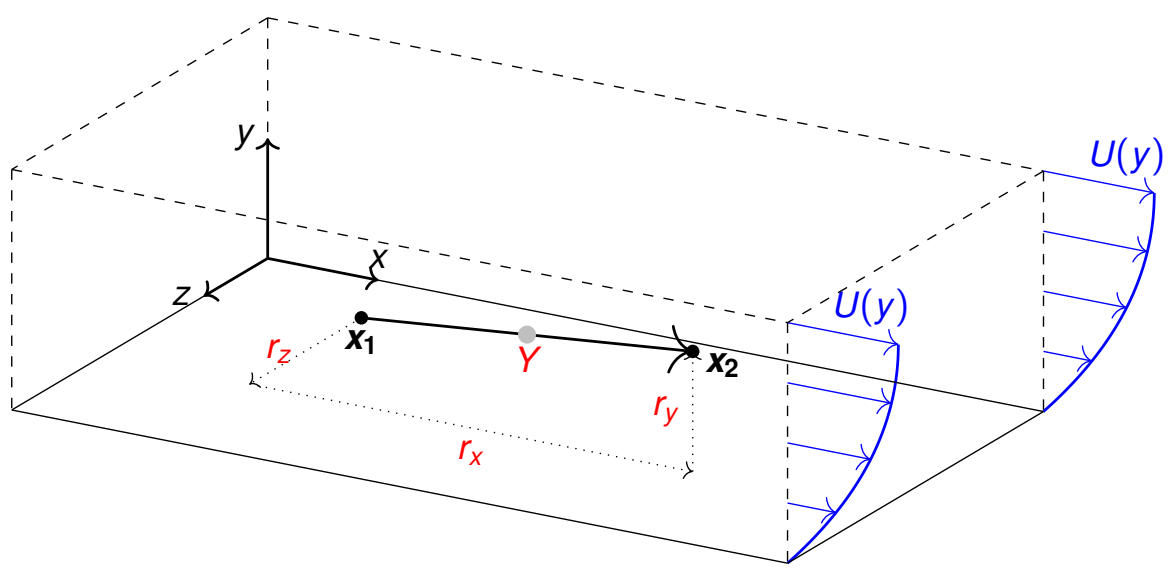




\section{Turbulent channel $\left(\operatorname{Re}_{\tau}=200\right):\langle\delta u \delta u\rangle$ in $r_{x}=0$ space}
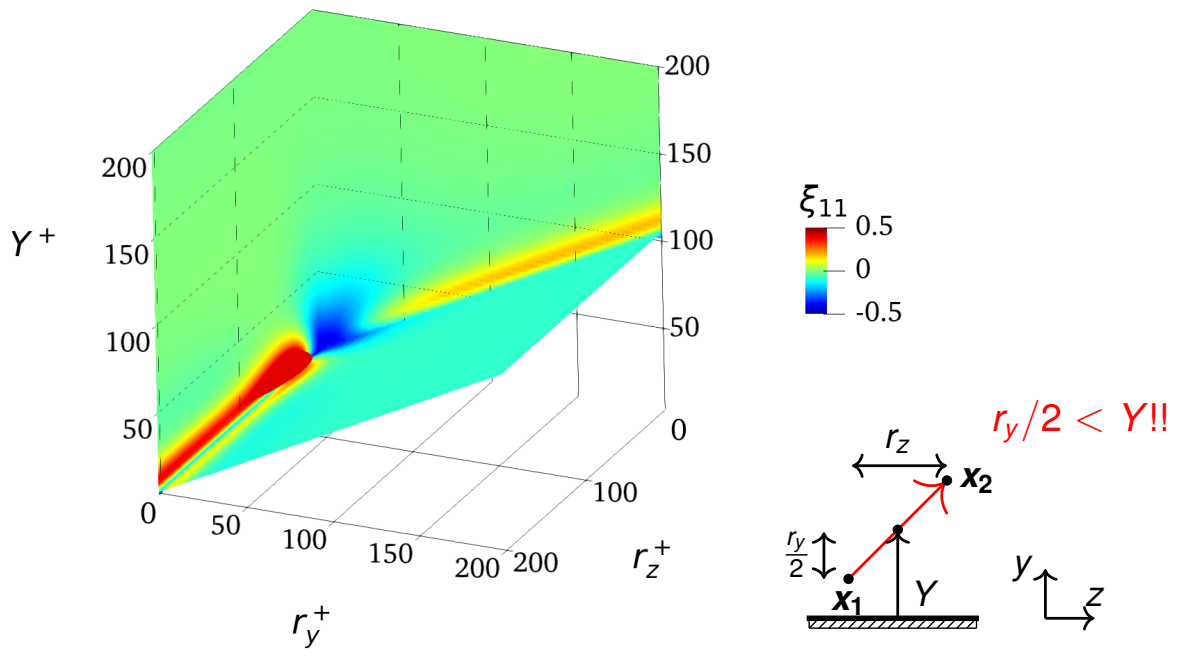


\section{Turbulent channel $\left(\operatorname{Re}_{\tau}=200\right):\langle\delta u \delta u\rangle$ in $r_{x}=0$ space}
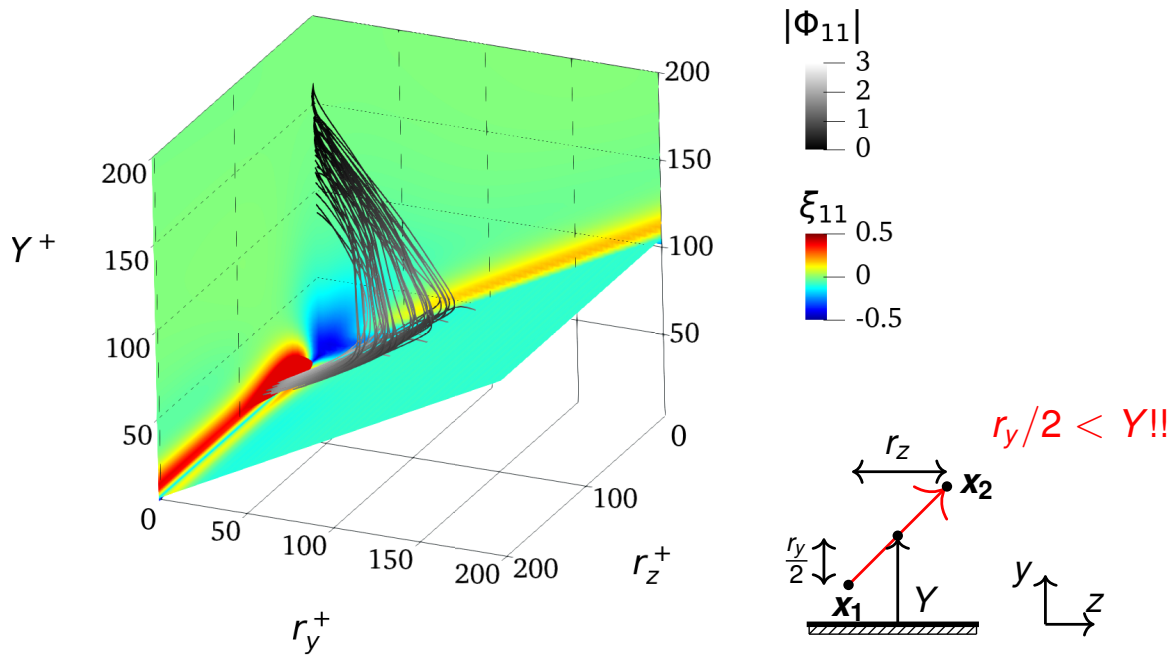


\section{$\langle\delta u \delta u\rangle$}

\section{versus}

$\left\langle\delta u_{i} \delta u_{i}\right\rangle$
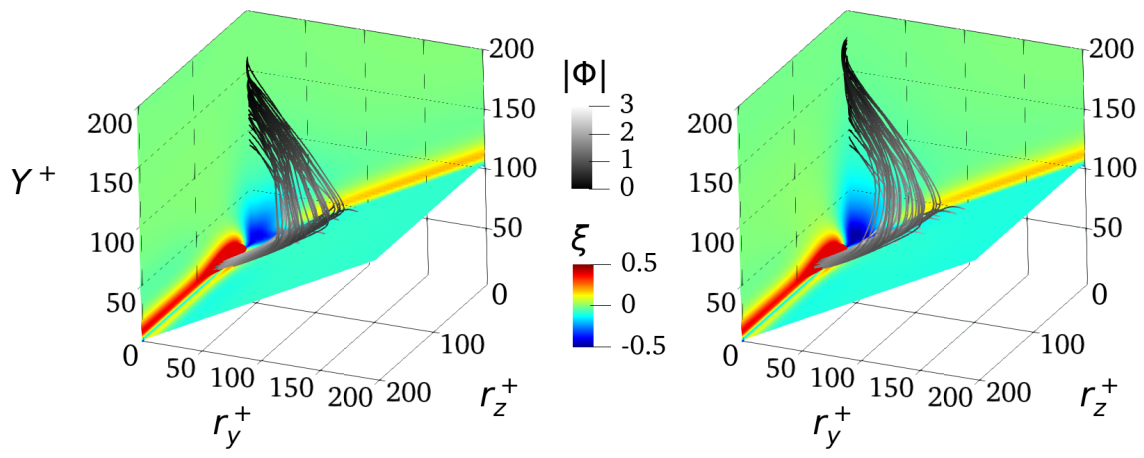


\section{$\langle\delta u \delta u\rangle$}

\section{versus}

$\left\langle\delta u_{i} \delta u_{i}\right\rangle$

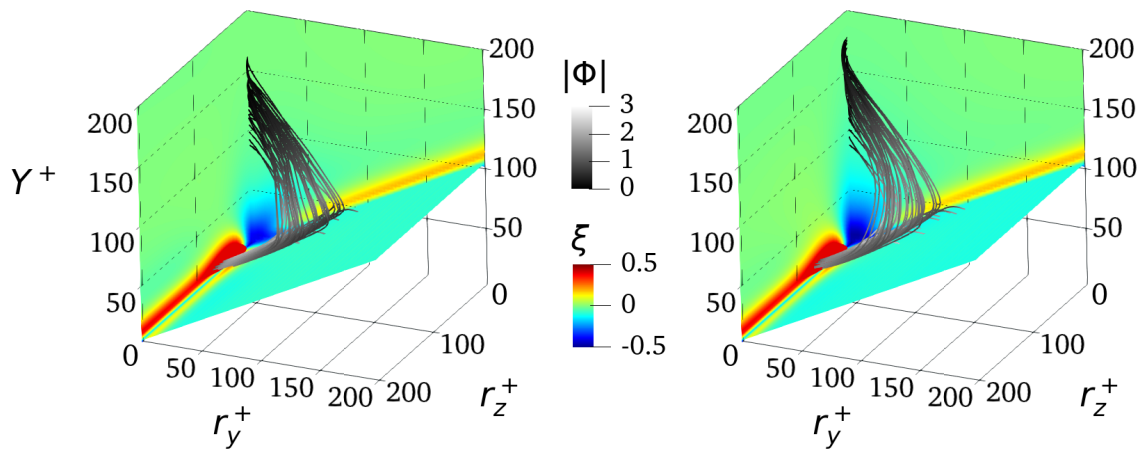

$\langle\delta u \delta u\rangle \approx\left\langle\delta u_{i} \delta u_{i}\right\rangle$ 


\section{$\langle\delta u \delta u\rangle$}

\section{versus}

$\left\langle\delta u_{i} \delta u_{i}\right\rangle$
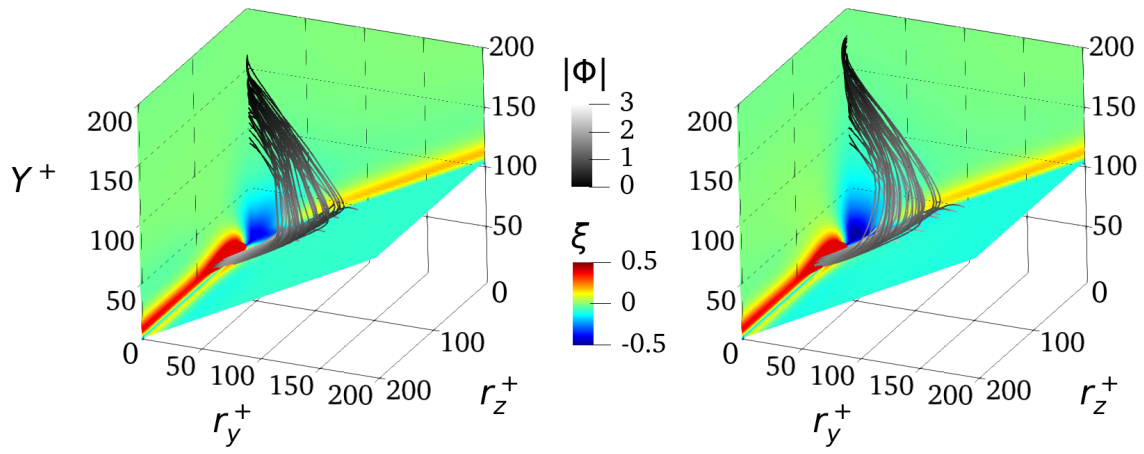

\section{$\langle\delta u \delta u\rangle \approx\left\langle\delta u_{i} \delta u_{i}\right\rangle$ \\ what does AGKE add to GKE?}




\section{Anisotropy}

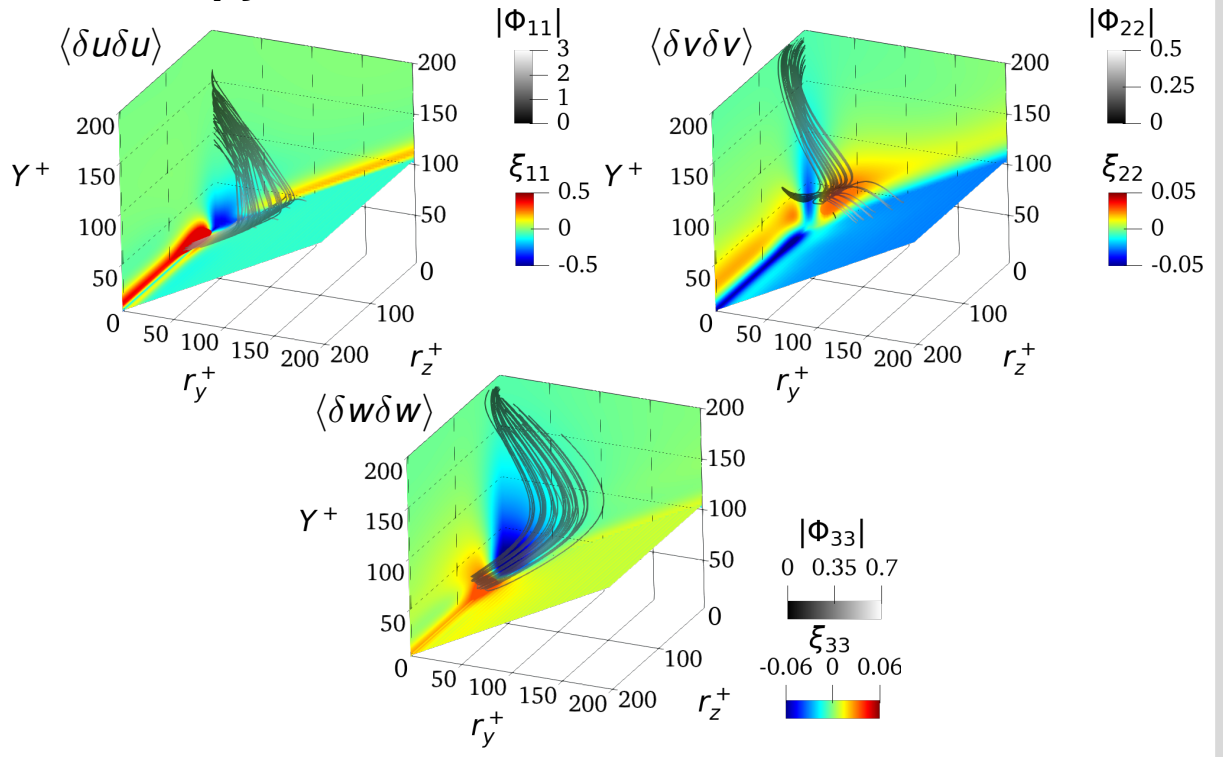




\section{Anisotropy}
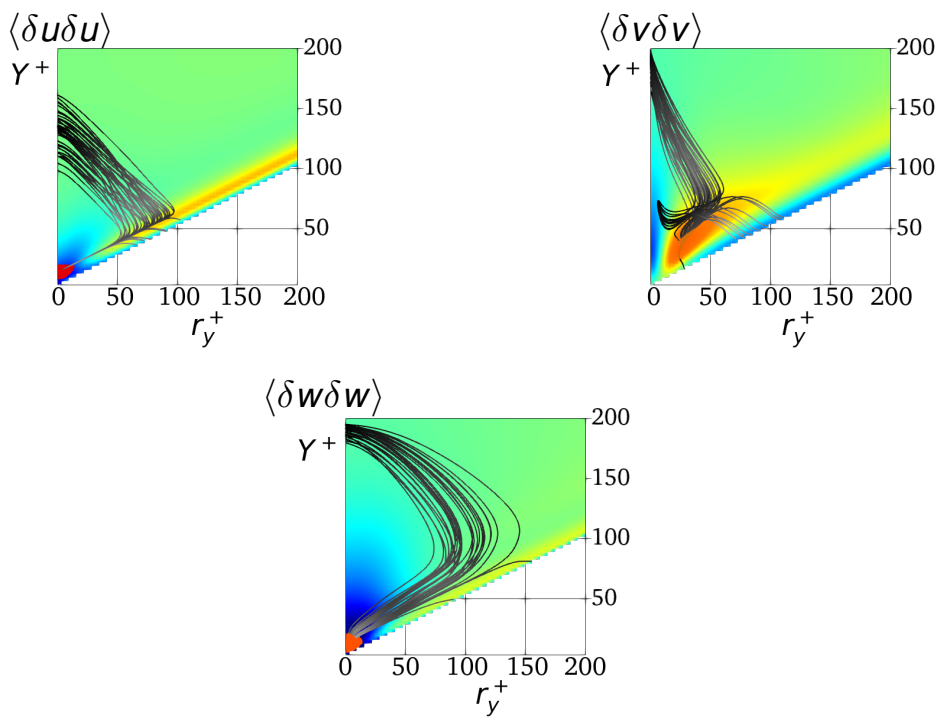


\section{Anisotropy of attached scales}
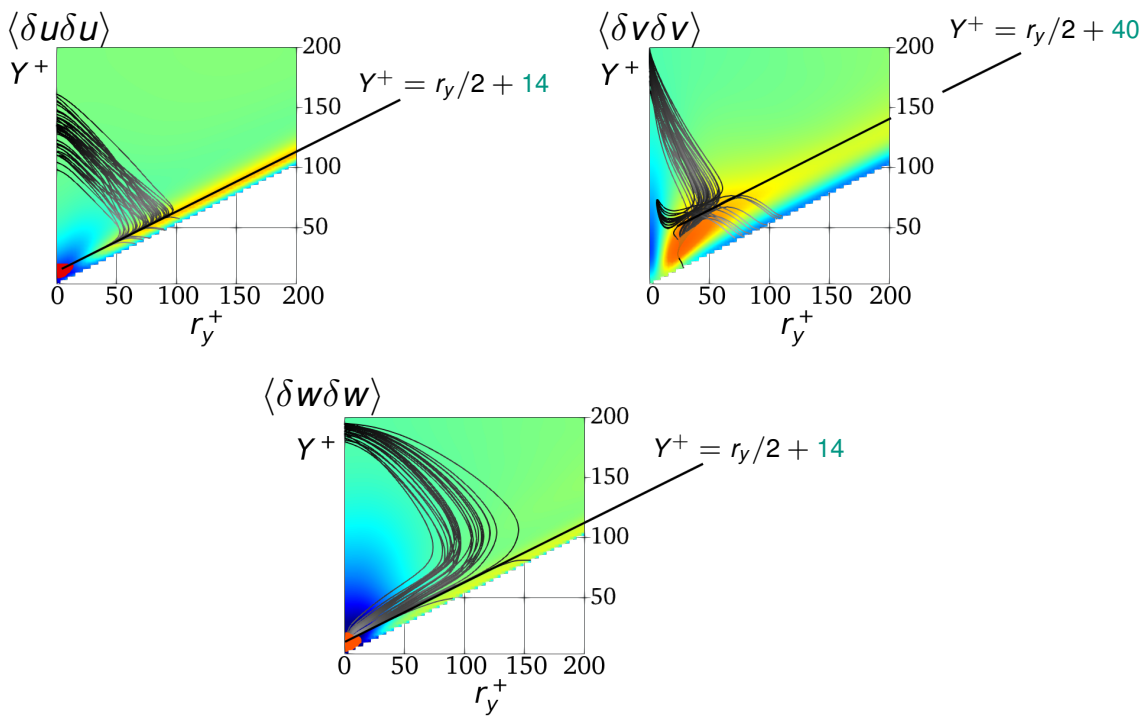


\section{Redistribution: pressure strain}
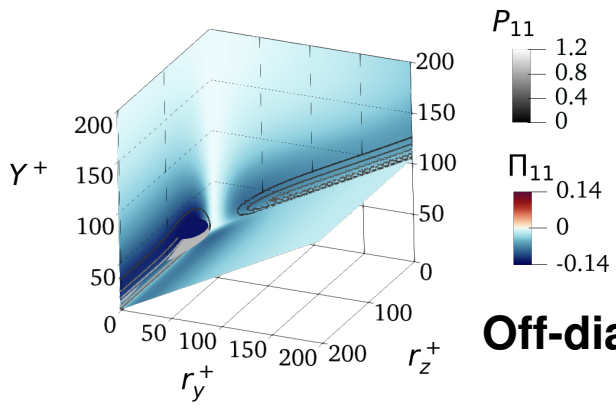

\section{Off-diagonal component $\langle\delta u \delta v\rangle$}

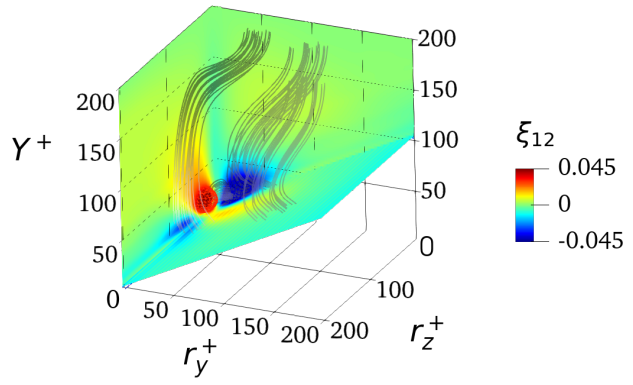




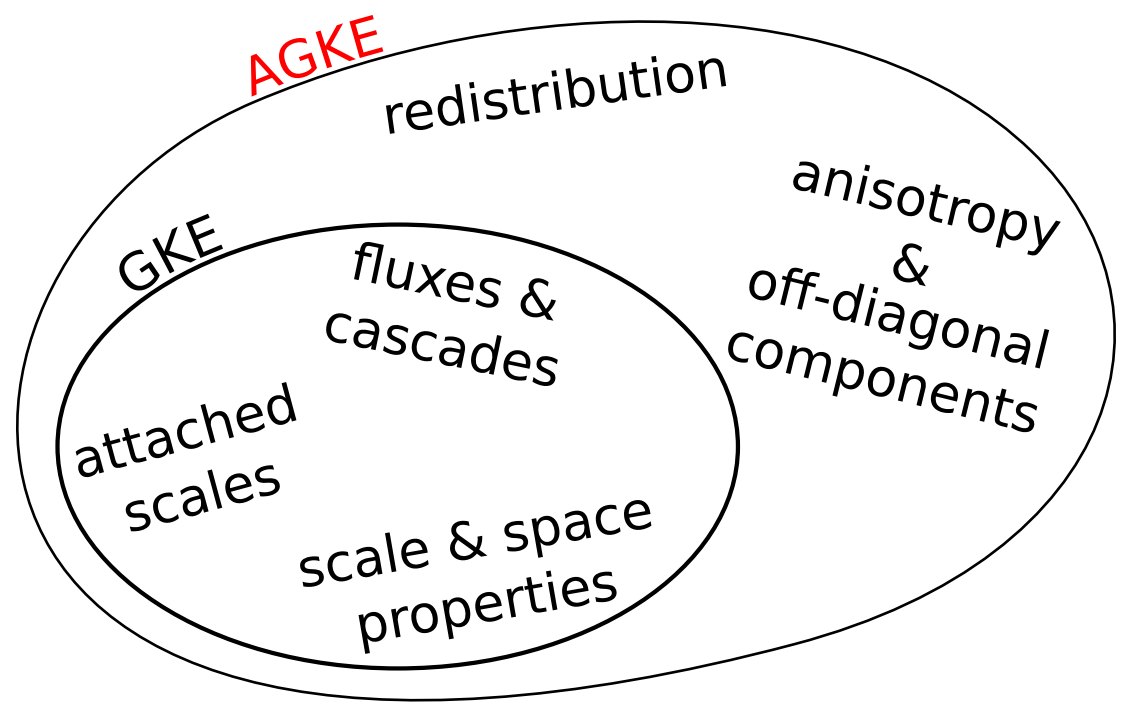




\section{How will I use the AGKE?}

Role and occurrence of large scales

- Very Large Scale Motions at high Re

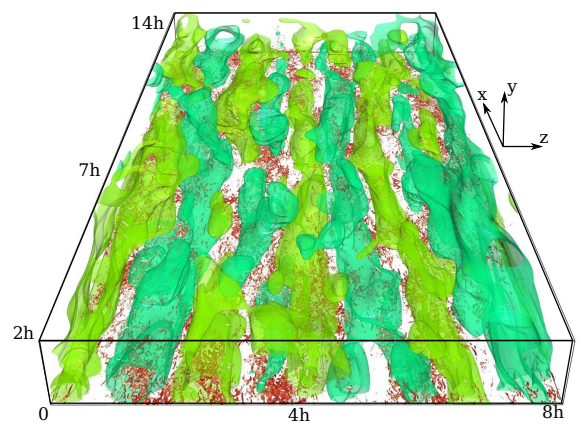

Gatti et al. FTaC 2018 


\section{How will I use the AGKE?}

Role and occurrence of large scales

- Very Large Scale Motions at high Re

- Secondary Motions of Prandtl second kind
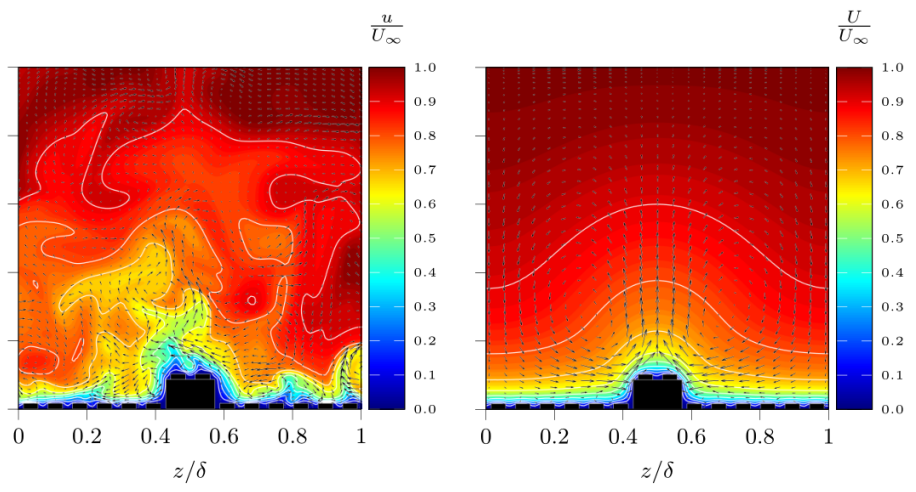

Stroh et al. JFM submitted 


\section{THANKS \\ for your kind attention!}

for questions and suggestions:

davide.gatti@kit.edu maurizio.quadrio@polimi.it alessandro.chiarini@polimi.it andrea.cimarelli@unimore.it 


\section{European Drag Reduction and Flow Control Meeting}

26-29 March 2019, Bad Herrenalb, Germany

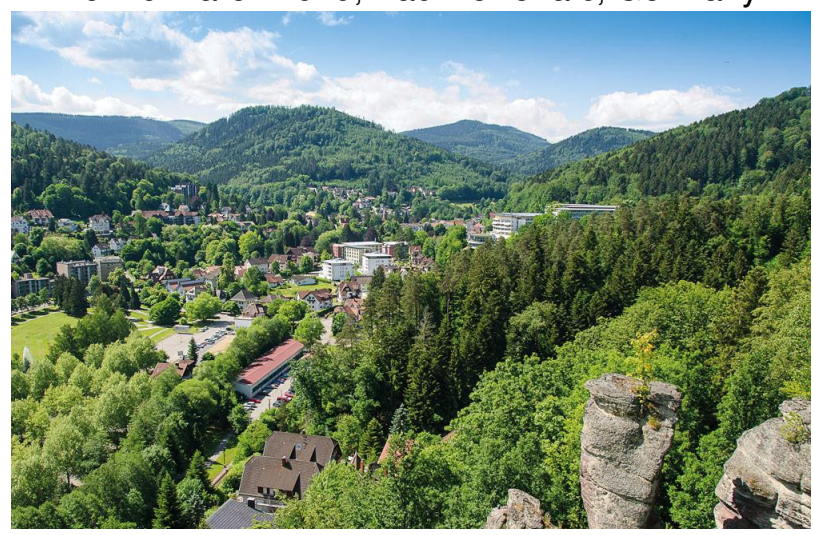

www.edrfcm.science 


\section{Conclusion}

We presented the AGKE: exact budget equations for $\left\langle\delta u_{i} \delta u_{j}\right\rangle$

a anisotropy

off-diagonal components

- redistribution

a In addition to spectral Reynolds stress budgets:

- no need for homogeneity

a allows scales in inhomogeneous directions

a possible "fluxes" interpretation

a probably interesting for your research too! 


\section{Conclusion}

We presented the AGKE: exact budget equations for $\left\langle\delta u_{i} \delta u_{j}\right\rangle$

- In addition to GKE:

- anisotropy

- off-diagonal components

- redistribution 


\section{Conclusion}

We presented the AGKE: exact budget equations for $\left\langle\delta u_{i} \delta u_{j}\right\rangle$

- In addition to GKE:

- anisotropy

- off-diagonal components

- redistribution

- In addition to spectral Reynolds stress budgets:

- no need for homogeneity

- allows scales in inhomogeneous directions

- possible "fluxes" interpretation 


\section{Conclusion}

We presented the AGKE: exact budget equations for $\left\langle\delta u_{i} \delta u_{j}\right\rangle$

- In addition to GKE:

- anisotropy

- off-diagonal components

- redistribution

- In addition to spectral Reynolds stress budgets:

- no need for homogeneity

- allows scales in inhomogeneous directions

- possible "fluxes" interpretation

- probably interesting for your research too! 


\section{Fluxes, field lines}
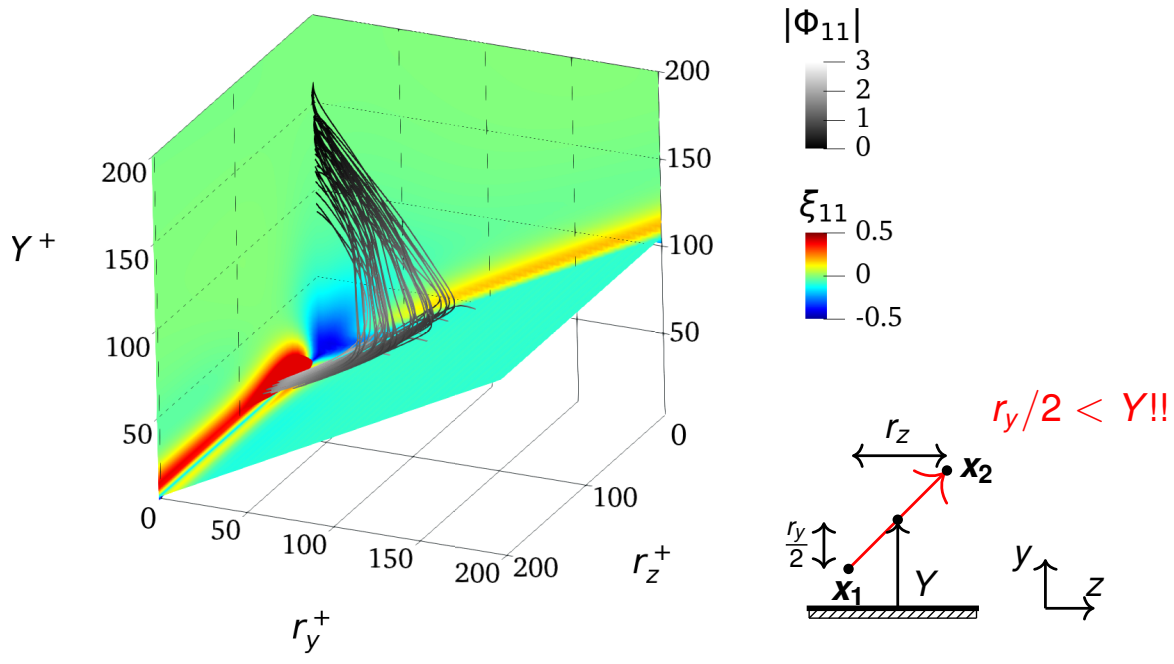


\section{Fluxes, field lines}

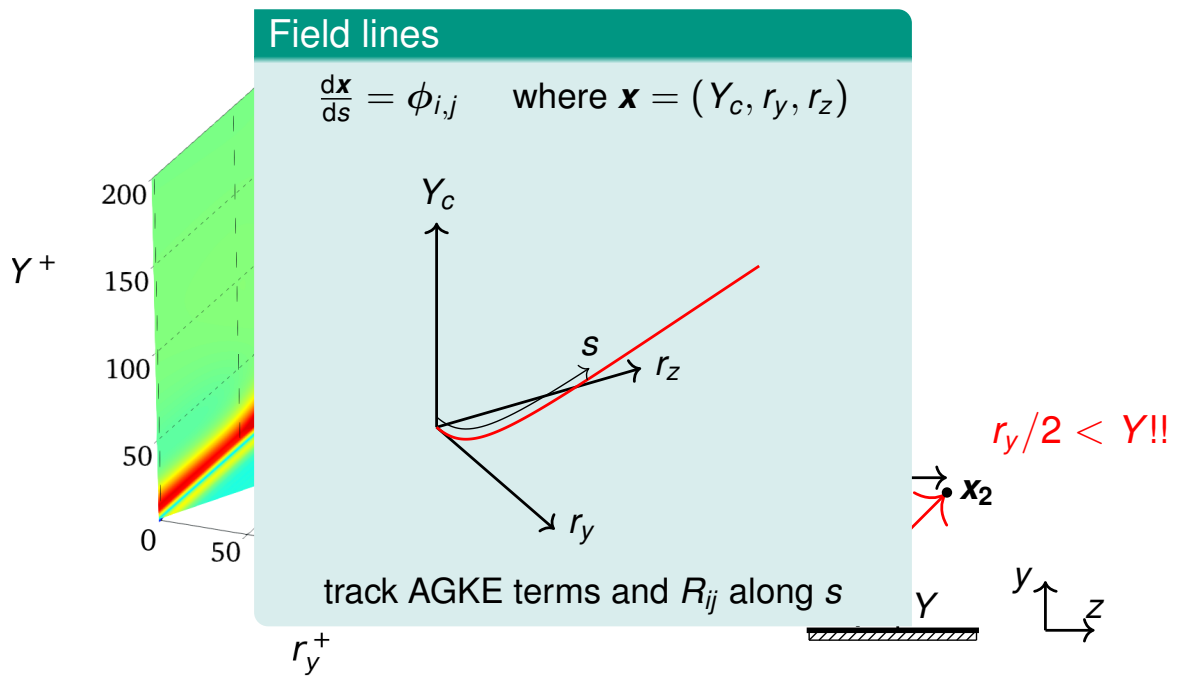




\section{Fluxes, field lines: attached \& detached scales}
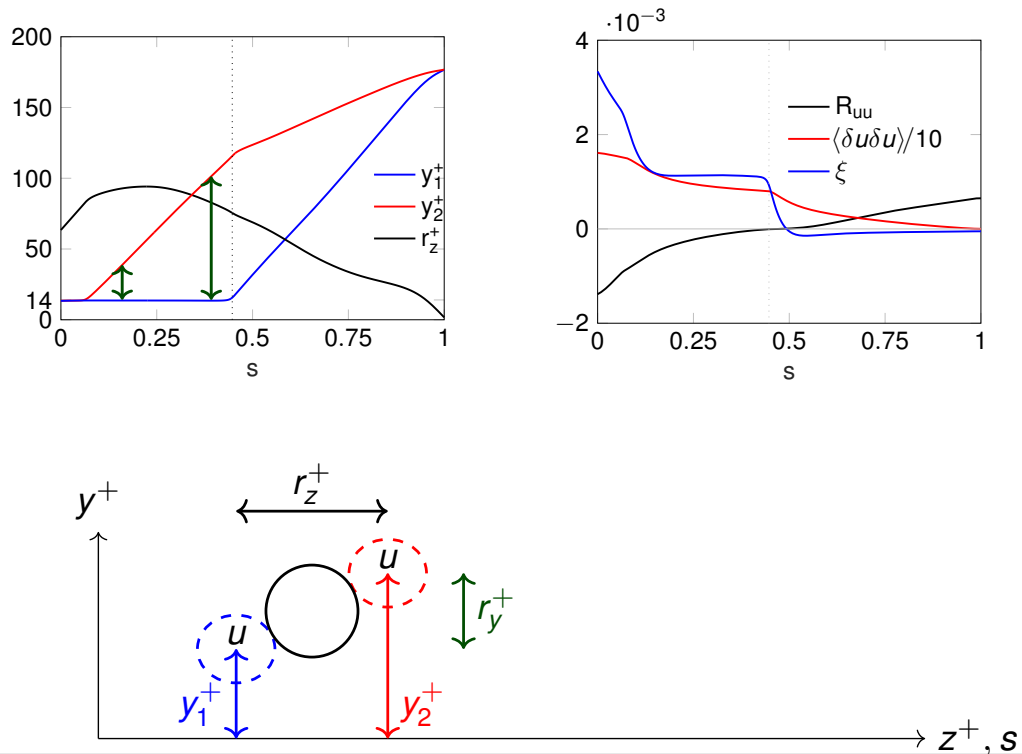


\section{Fluxes, field lines: attached \& detached scales}
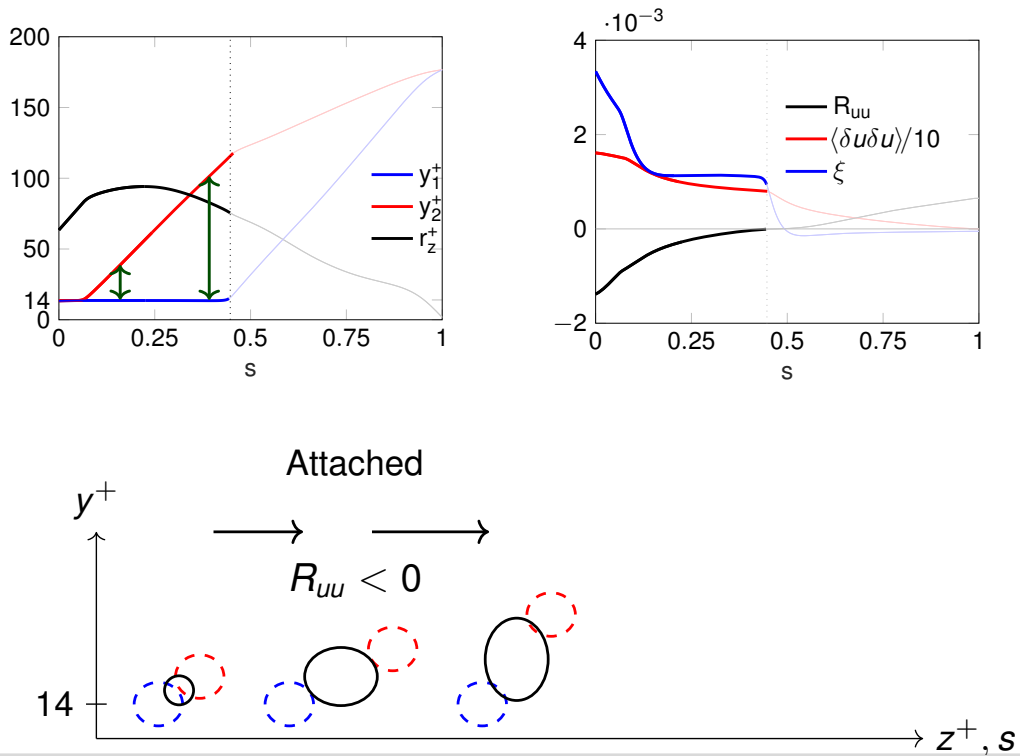


\section{Fluxes, field lines: attached \& detached scales}
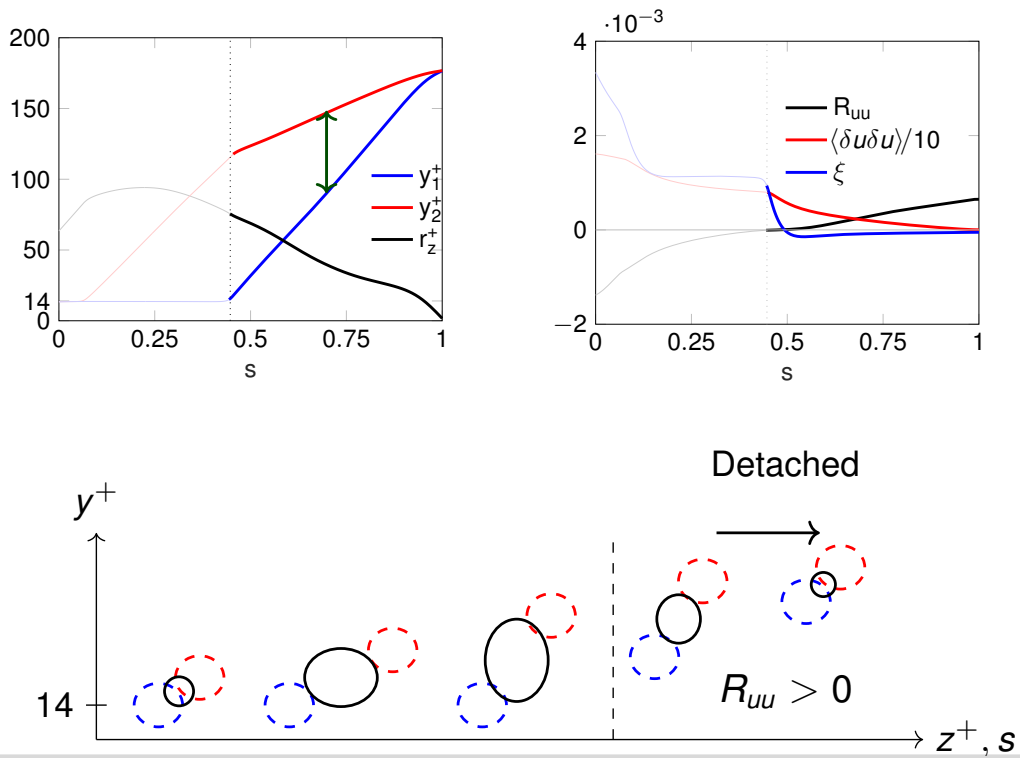


\section{Fluxes, divergence: donor \& receiver scales}

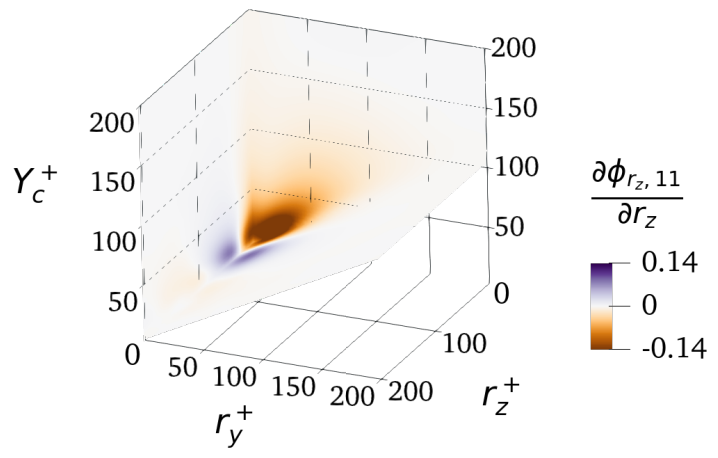

contribution of various physical processes to $\left\langle\delta u_{i} \delta u_{j}\right\rangle$

(e.g. nonlinear turbulent transport) 


\section{$\left\langle\delta u_{i} \delta u_{j}\right\rangle:$ relationship with correlation $R_{i} j$}

$$
\left\langle\delta u_{i} \delta u_{j}\right\rangle\left(Y_{c}, r_{x}, r_{y}, r_{z}\right)=\underbrace{\left.\left\langle u_{i} u_{j}\right\rangle\right|_{y_{1}}+\left.\left\langle u_{i} u_{j}\right\rangle\right|_{y_{2}}}_{\text {sum of variances }}-\underbrace{2 R_{u_{i}\left|y_{1} u_{j}\right|_{y_{2}}}\left(r_{x}, r_{z}\right)}_{\text {Cross-correlation }}
$$




\section{$\left\langle\delta u_{i} \delta u_{j}\right\rangle:$ relationship with correlation $R_{i} j$}

$$
\left\langle\delta u_{j} \delta u_{j}\right\rangle\left(Y_{c}, r_{x}, r_{y}, r_{z}\right)=\underbrace{\left.\left\langle u_{i} u_{j}\right\rangle\right|_{y_{1}}+\left.\left\langle u_{i} u_{j}\right\rangle\right|_{y_{2}}}_{\text {sum of variances }}-\underbrace{2 R_{u_{i} y_{1} u_{j} \mid y_{2}}\left(r_{x}, r_{z}\right)}_{\text {Cross-correlation }}
$$

$$
\begin{aligned}
& y_{1}=Y_{c}-r_{y} / 2 \\
& y_{2}=Y_{c}+r_{y} / 2
\end{aligned}
$$

$\left(Y_{c}, r_{x}, r_{y}, r_{z}\right) \leftrightarrow\left(y_{1}, y_{2}, r_{x}, r_{z}\right)$

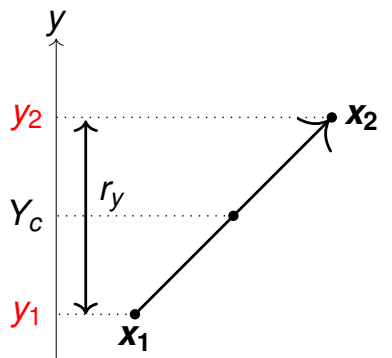

$x(z)$ 


\section{$\left\langle\delta u_{i} \delta u_{j}\right\rangle:$ relationship with correlation $R_{i} j$}

\section{$\left\langle\delta u_{i} \delta u_{j}\right\rangle\left(Y_{c}, r_{x}, r_{y}, r_{z}\right)=\underbrace{\left.\left\langle u_{i} u_{j}\right\rangle\right|_{y_{1}}+\left.\left\langle u_{i} u_{j}\right\rangle\right|_{y_{2}}}_{\text {sum of variances }}-\underbrace{2 R_{u_{i}\left|y_{1} u_{j}\right|_{y_{2}}}\left(r_{x}, r_{z}\right)}_{\text {Cross-correlation }}$}

$\left\langle\delta u_{i} \delta u_{j}\right\rangle\left(Y_{c}, r_{x}, r_{y}, r_{z}\right) \neq\left.\left\langle u_{i} u_{j}\right\rangle\right|_{y_{1}}+\left.\left\langle u_{i} u_{j}\right\rangle\right|_{y_{2}}$

$$
R_{\left.\left.u_{i}\right|_{y_{1}} u_{j}\right|_{y_{2}}}\left(r_{x}, r_{z}\right) \neq 0
$$

\section{Coherent structures!}

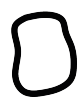




\section{$\left\langle\delta u_{i} \delta u_{j}\right\rangle:$ relationship with correlation $R_{i} j$}

$$
\langle\delta u \delta u\rangle\left(Y_{c}, 0, r_{y}, r_{z}\right)>\underbrace{\left.\langle u u\rangle\right|_{y_{1}}+\left.\langle u u\rangle\right|_{y_{2}}}_{\text {sum of variances }}-\underbrace{2 R_{\left.\left.u\right|_{y_{1}} u\right|_{y_{2}}}\left(0, r_{z}\right)}_{\text {Cross-correlation }}
$$




\section{$\left\langle\delta u_{i} \delta u_{j}\right\rangle:$ relationship with correlation $R_{i} j$}

$$
\langle\delta u \delta u\rangle\left(Y_{c}, 0, r_{y}, r_{z}\right)=\underbrace{\left.\langle u u\rangle\right|_{y_{1}}+\left.\langle u u\rangle\right|_{y_{2}}}_{\text {sum of variances }}-\underbrace{2 R_{\left.\left.u\right|_{y_{1}} u\right|_{y_{2}}}\left(0, r_{z}\right)}_{\text {Cross-correlation }}<0
$$

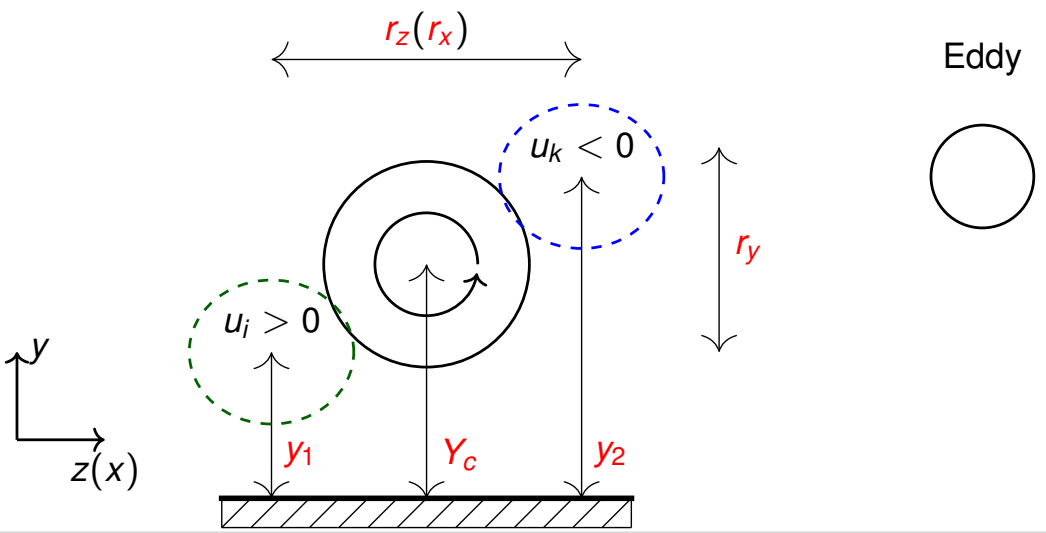




\section{$\left\langle\delta u_{i} \delta u_{j}\right\rangle:$ relationship with correlation $R_{i} j$}

$$
\left\langle\delta u_{i} \delta u_{j}\right\rangle\left(Y_{c}, r_{x}, r_{y}, r_{z}\right) \leftrightarrow \underbrace{\left.\left\langle u_{i} u_{j}\right\rangle\right|_{y_{1}}+\left.\left\langle u_{i} u_{j}\right\rangle\right|_{y_{2}}}_{\text {sum of variances }}-\underbrace{2 R_{\left.\left.u_{i}\right|_{y_{1}} u_{j}\right|_{y_{2}}\left(r_{x}, r_{z}\right)}}_{\text {Cross-correlation }}
$$

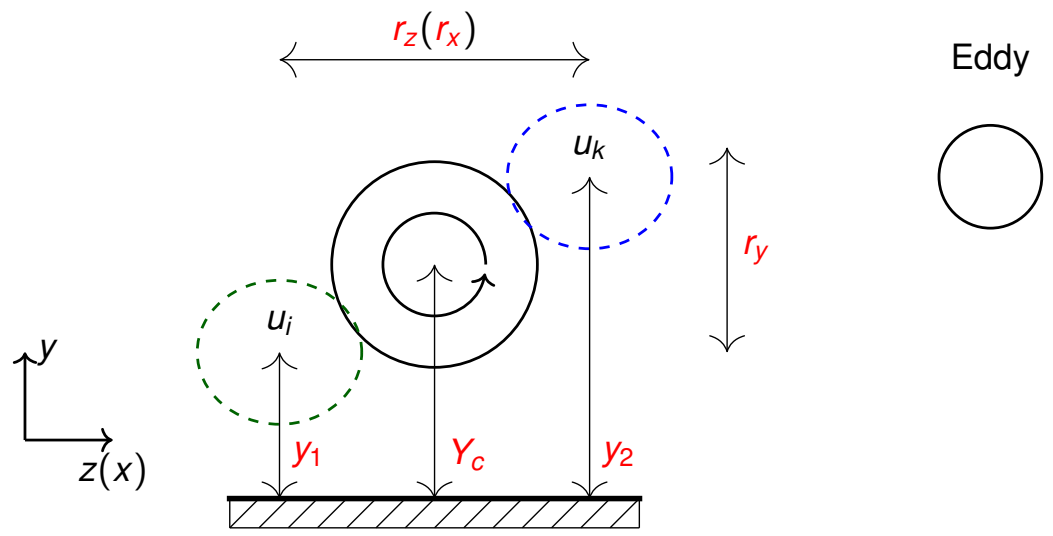

